\title{
EDGE DETECTION TECHNIQUES FOR IMAGE SEGMENTATION
}

\author{
Muthukrishnan. $\mathrm{R}^{1}$ and M.Radha ${ }^{2}$ \\ ${ }^{1}$ Assistant Professor, Department of Statistics, Bharathiar University, Coimbatore. \\ muthukrishnan70@rediffmail.com \\ ${ }^{2}$ Research Scholar, Department of Statistics, Bharathiar University, Coimbatore. \\ radhamyilsamy@gmail. com
}

\begin{abstract}
Interpretation of image contents is one of the objectives in computer vision specifically in image processing. In this era it has received much awareness of researchers. In image interpretation the partition of the image into object and background is a severe step. Segmentation separates an image into its component regions or objects. Image segmentation t needs to segment the object from the background to read the image properly and identify the content of the image carefully. In this context, edge detection is a fundamental tool for image segmentation. In this paper an attempt is made to study the performance of most commonly used edge detection techniques for image segmentation and also the comparison of these techniques is carried out with an experiment by using MATLAB software.
\end{abstract}

\section{Keywords}

Computer Vision, Image Segmentation, Edge detection, MATLAB.

\section{INTRODUCTION}

Image segmentation is an essential step in image analysis. Segmentation separates an image into its component parts or objects. The level to which the separation is carried depends on the problem being solved. When the objects of interest in an application have been inaccessible the segmentation must stop. Segmentation algorithms for images generally based on the discontinuity and similarity of image intensity values. Discontinuity approach is to partition an image based on abrupt changes in intensity and similarity is based on partitioning an image into regions that are similar according to a set of predefined criteria. Thus the choice of image segmentation technique is depends on the problem being considered. Edge detection is a part of image segmentation. The effectiveness of many image processing also computer vision tasks depends on the perfection of detecting meaningful edges. It is one of the techniques for detecting intensity discontinuities in a digital image.

The process of classifying and placing sharp discontinuities in an image is called the edge detection. The discontinuities are immediate changes in pixel concentration which distinguish boundaries of objects in a scene. Classical methods of edge detection engage convolving the image through an operator, which is constructed to be perceptive to large gradients in the image although returning values of zero in uniform regions. There is a very large amount of edge detection techniques available, each technique designed to be perceptive to certain types of edges. Variables concerned in the selection of an edge detection operator consist of Edge orientation, Edge structure and Noise environment. The geometry of the operator establishes a DOI : $10.5121 /$ ijcsit.2011.3620 
characteristic direction in which it is most perceptive to edges. Operators can be optimized to look for vertical, horizontal, or diagonal edges. Edge detection is a difficult task in noisy images, since both the edges and noise hold high- frequency content. Efforts to reduce the noise result in unclear and distorted edges. Techniques used on noisy images are typically larger in scope; therefore they can common enough data to discount localized noisy pixels. This results in less perfect localization of the detected edges. Not all edges involve a step change in intensity. Things such as refraction or reduced focus can result in objects through boundaries defined by a regular change in intensity. The method wants to be chosen to be receptive to such a regular change in those cases. So, there are some problems of fake edge detection, edge localization, missing true edges, problems due to noise and high computational time etc. Hence, the objective is to do the comparison of a variety of edge detections and analyze the performance of the different techniques in various conditions.

In this paper an attempt is made to review some of the most commonly used edge detection techniques for image segmentation and also performances of such techniques is carried out for an image by using MATLAB software. Section 2 introduces the basic concepts that are mostly employed in the literature. Section 3 provides a comprehensive theoretical and mathematical background for edge detection and explains different computing approaches to edge detection. Section 4 presents the comparison of various edge detection techniques with an image. Section 5 contains a quick discussion about the reviewed works as well as conclusion.

\section{IMAGE SEGMENTATION}

Image Segmentation is the process of partitioning a digital image into multiple regions or sets of pixels. Essentially, in image partitions are different objects which have the same texture or color. The image segmentation results are a set of regions that cover the entire image together and a set of contours extracted from the image. All of the pixels in a region are similar with respect to some characteristics such as color, intensity, or texture. Adjacent regions are considerably different with respect to the same individuality. The different approaches are (i) by finding boundaries between regions based on discontinuities in intensity levels, (ii) thresholds based on the distribution of pixel properties, such as intensity values, and (iii) based on finding the regions directly. Thus the choice of image segmentation technique is depends on the problem being considered.

Region based methods are based on continuity. These techniques divide the entire image into sub regions depending on some rules like all the pixels in one region must have the same gray level. Region-based techniques rely on common patterns in intensity values within a cluster of neighboring pixels. The cluster is referred to as the region in addition to group the regions according to their anatomical or functional roles are the goal of the image segmentation. Threshold is the simplest way of segmentation. Using thresholding technique regions can be classified on the basis range values, which is applied to the intensity values of the image pixels. Thresholding is the transformation of an input image to an output that is segmentated binary image. Segmentation Methods based on finding the regions directly find for abrupt changes in the intensity value. These methods are called as Edge or Boundary based methods. Edge detection is the problem of fundamental importance in image analysis. Edge detection techniques are generally used for finding discontinuities in gray level images. To detect consequential discontinuities in the gray level image is the important common approach in edge detection. Image segmentation methods for detecting discontinuities are boundary based methods. 


\section{EDGE DETECTION TECHNIQUES}

The edge representation of an image significantly reduces the quantity of data to be processed, yet it retains essential information regarding the shapes of objects in the scene. This explanation of an image is easy to incorporate into a large amount of object recognition algorithms used in computer vision along with other image processing applications. The major property of the edge detection technique is its ability to extract the exact edge line with good orientation as well as more literature about edge detection has been available in the past three decades. On the other hand, there is not yet any common performance directory to judge the performance of the edge detection techniques. The performance of an edge detection techniques are always judged personally and separately dependent to its application.

Edge detection is a fundamental tool for image segmentation. Edge detection methods transform original images into edge images benefits from the changes of grey tones in the image. In image processing especially in computer vision, the edge detection treats the localization of important variations of a gray level image and the detection of the physical and geometrical properties of objects of the scene. It is a fundamental process detects and outlines of an object and boundaries among objects and the background in the image. Edge detection is the most familiar approach for detecting significant discontinuities in intensity values.

Edges are local changes in the image intensity. Edges typically occur on the boundary between two regions. The main features can be extracted from the edges of an image. Edge detection has major feature for image analysis. These features are used by advanced computer vision algorithms. Edge detection is used for object detection which serves various applications like medical image processing, biometrics etc. Edge detection is an active area of research as it facilitates higher level image analysis. There are three different types of discontinuities in the grey level like point, line and edges. Spatial masks can be used to detect all the three types of discontinuities in an image.

There are many edge detection techniques in the literature for image segmentation. The most commonly used discontinuity based edge detection techniques are reviewed in this section. Those techniques are Roberts edge detection, Sobel Edge Detection, Prewitt edge detection, Kirsh edge detection, Robinson edge detection, Marr-Hildreth edge detection, LoG edge detection and Canny Edge Detection.

\subsection{Roberts Edge Detection}

The Roberts edge detection is introduced by Lawrence Roberts (1965). It performs a simple, quick to compute, 2-D spatial gradient measurement on an image. This method emphasizes regions of high spatial frequency which often correspond to edges. The input to the operator is a grayscale image the same as to the output is the most common usage for this technique. Pixel values in every point in the output represent the estimated complete magnitude of the spatial gradient of the input image at that point.

\begin{tabular}{|c|c|}
\hline-1 & 0 \\
\hline 0 & +1 \\
\hline \multicolumn{2}{|c|}{$\mathrm{G}_{\mathrm{X}}$}
\end{tabular}

\begin{tabular}{|c|c|}
\hline 0 & -1 \\
\hline+1 & 0 \\
\hline \multicolumn{2}{|c}{$\mathrm{G}_{\mathrm{y}}$}
\end{tabular}




\subsection{Sobel Edge Detection}

The Sobel edge detection method is introduced by Sobel in 1970 (Rafael C.Gonzalez (2004)). The Sobel method of edge detection for image segmentation finds edges using the Sobel approximation to the derivative. It precedes the edges at those points where the gradient is highest. The Sobel technique performs a 2-D spatial gradient quantity on an image and so highlights regions of high spatial frequency that correspond to edges. In general it is used to find the estimated absolute gradient magnitude at each point in $n$ input grayscale image. In conjecture at least the operator consists of a pair of $3 \times 3$ complication kernels as given away in under table. One kernel is simply the other rotated by $90^{\circ}$. This is very alike to the Roberts Cross operator.

\begin{tabular}{|c|c|c|}
\hline-1 & -2 & -1 \\
\hline 0 & 0 & 0 \\
\hline+1 & +2 & +1 \\
\hline
\end{tabular}

$\mathrm{G}_{\mathrm{x}}$

\begin{tabular}{|c|c|c|}
\hline-1 & 0 & -1 \\
\hline-2 & 0 & +2 \\
\hline-1 & 0 & +1 \\
\hline
\end{tabular}

$\mathrm{G}_{\mathrm{y}}$

\subsection{Prewitt Edge Detection}

The Prewitt edge detection is proposed by Prewitt in 1970 (Rafael C.Gonzalez [1]. To estimate the magnitude and orientation of an edge Prewitt is a correct way. Even though different gradient edge detection wants a quite time consuming calculation to estimate the direction from the magnitudes in the $\mathrm{x}$ and $\mathrm{y}$-directions, the compass edge detection obtains the direction directly from the kernel with the highest response. It is limited to 8 possible directions; however knowledge shows that most direct direction estimates are not much more perfect. This gradient based edge detector is estimated in the $3 \times 3$ neighborhood for eight directions. All the eight convolution masks are calculated. One complication mask is then selected, namely with the purpose of the largest module.

\begin{tabular}{|c|c|c|}
\hline-1 & -1 & -1 \\
\hline 0 & 0 & 0 \\
\hline+1 & +1 & +1 \\
\hline
\end{tabular}

$\mathrm{G}_{\mathrm{x}}$

\begin{tabular}{|l|l|l|}
\hline-1 & 0 & +1 \\
\hline-1 & 0 & +1 \\
\hline-1 & 0 & +1 \\
\hline
\end{tabular}

$\mathrm{G}_{\mathrm{y}}$

Prewitt detection is slightly simpler to implement computationally than the Sobel detection, but it tends to produce somewhat noisier results.

\subsection{Kirsch Edge detection}

Kirsch edge detection is introduced by Kirsch (1971). The masks of this Kirsch technique are defined by considering a single mask and rotating it to eight main compass directions: North, Northwest, West, Southwest, South, Southeast, East and Northeast. The masks are distinct as follows: 
International Journal of Computer Science \& Information Technology (IJCSIT) Vol 3, No 6, Dec 2011

$$
\begin{aligned}
& E=\left[\begin{array}{ccc}
-3 & -3 & 5 \\
-3 & 0 & 5 \\
-3 & -3 & 5
\end{array}\right] \quad N E=\left[\begin{array}{ccc}
-3 & 5 & 5 \\
-3 & 0 & 5 \\
-3 & -3 & -3
\end{array}\right] N=\left[\begin{array}{ccc}
5 & 5 & 5 \\
-3 & 0 & -3 \\
-3 & -3 & -3
\end{array}\right] \quad N W=\left[\begin{array}{ccc}
5 & \mathrm{k}_{3} & -3 \\
5 & 0 & -3 \\
-3 & -3 & -3
\end{array}\right] \\
& W=\left[\begin{array}{ccc}
5 & -3 & -3 \\
5 & 0 & -3 \\
5 & -3 & -3
\end{array}\right] \quad S W=\left[\begin{array}{ccc}
-3 & -3 & -3 \\
5 & 0 & -3 \\
5 & 5 & -3
\end{array}\right] \quad S=\left[\begin{array}{ccc}
-3 & -3 & -3 \\
-3 & 0 & -3 \\
5 & 5 & 5
\end{array}\right] \quad S E=\left[\begin{array}{ccc}
-3 & -3 & 5 \\
-3 & 0 & 5 \\
-3 & 5 & 5
\end{array}\right]
\end{aligned}
$$

The edge magnitude is defined as the maximum value found by convolution of each mask with the image. The direction is defined by mask that produces the maximum magnitude. Example, mask $\mathrm{k}_{0}$ corresponds to a vertical edge, while mask $\mathrm{k}_{5}$ corresponds to a diagonal edge. Notice that the last four masks are actually the same as the first four, but flipped about a central axis.

\subsection{Robinson Edge detection}

The Robinson method (Robinson 1977) is similar to Kirsch masks but is easier to implement because they rely only on coefficients of 0,1 and 2 . The masks are symmetrical about their directional axis, the axis with the zeros. One need only to compute the result on four masks and the result from other four can be obtained by negating the result from the first four. The masks are as follows:

$$
\begin{aligned}
& E=\left[\begin{array}{lll}
-1 & 0 & 1 \\
-2 & 0 & 2 \\
-1 & 0 & 1
\end{array}\right] \quad N E=\left[\begin{array}{ccc}
0 & 1 & 2 \\
-1 & 0 & 1 \\
-2 & -1 & 0
\end{array}\right] \quad N=\left[\begin{array}{ccc}
1 & 2 & 1 \\
0 & 0 & 0 \\
-1 & -2 & -1
\end{array}\right] \quad N W=\left[\begin{array}{ccc}
\mathrm{r}_{0} & \mathrm{r}_{2} \\
1 & 1 & 0 \\
1 & 0 & -1 \\
0 & -1 & -2
\end{array}\right] \\
& W=\left[\begin{array}{ccc}
1 & 0 & -1 \\
2 & 0 & -2 \\
1 & 0 & -1
\end{array}\right] \quad S W=\left[\begin{array}{ccc}
0 & -1 & -2 \\
1 & 0 & -1 \\
2 & 1 & 0
\end{array}\right] \quad S=\left[\begin{array}{ccc}
-1 & -2 & -1 \\
0 & 0 & 0 \\
1 & 2 & 1
\end{array}\right] \quad S E=\left[\begin{array}{ccc}
-2 & -1 & 0 \\
-1 & 0 & 1 \\
0 & 1 & 2
\end{array}\right]
\end{aligned}
$$

The magnitude of the gradient is the maximum value gained from applying all eight masks to the pixel neighborhood, and the angle of the gradient can be approximated as the angle of the line of zeroes in the mask yielding the maximum response.

\subsection{Marr-Hildreth Edge Detection}

The Marr-Hildreth (1980) technique is a method of detecting edges in digital images that is continuous curves wherever there are well-built and fast variations in image brightness. It is a easy and it operates by convolving the image with the LoG function, or, as a quick approximation by DoGs. Subsequently the zero-crossings are discovered in the filtered result to find the edges. The LoG method is sometimes as well referred to as the Mexican hat wavelet due to its image shape while turned up-side-down. Algorithm for the Marr-Hildreth edge detector is: 
- Smooth the image using a Gaussian

- Apply a two-dimensional Laplacian to the smoothed image (often the first two steps are combined into a single operation)

- Loop through the result and look for sign changes. If there is a sign change plus the slope across the sign change is greater than some threshold, mark as an edge.

- To get better results it is possible to run the result of the Laplacian through a hysteresis alike to Canny's edge detection although this is not how the edge detector was firstly implemented.

\subsection{LoG edge detection}

The Laplacian of Gaussian (LoG) was proposed by Marr(1982). The LoG of an image $f(x, y)$ is a second order derivative defined as,

$$
\nabla^{2} f=\frac{\partial^{2} f}{\partial x^{2}}+\frac{\partial^{2} f}{\partial y^{2}}
$$

It has two effects, it smoothes the image and it computes the Laplacian, whch yields a doubleedge image. Locating edges then consists of finding the zero crossings between the double edges. The digital implementation of the Laplacian function is usually made through the mask below,

\begin{tabular}{|c|c|c|}
\hline 0 & -1 & 0 \\
\hline-1 & 4 & -1 \\
\hline 0 & -1 & 0 \\
\hline
\end{tabular}

$\mathrm{G}_{\mathrm{x}}$

\begin{tabular}{|c|c|c|}
\hline-1 & -1 & -1 \\
\hline-1 & 8 & -1 \\
\hline-1 & -1 & -1 \\
\hline
\end{tabular}

$\mathrm{G}_{\mathrm{y}}$

The Laplacian is generally used to found whether a pixel is on the dark or light side of an edge.

\subsection{Canny Edge Detection}

In industry, the Canny edge detection technique is one of the standard edge detection techniques. It was first created by John Canny for his Master's thesis at MIT in 1983, and still outperforms many of the newer algorithms that have been developed. To find edges by separating noise from the image before find edges of image the Canny is a very important method. Canny method is a better method without disturbing the features of the edges in the image afterwards it applying the tendency to find the edges and the serious value for threshold. The algorithmic steps are as follows:

- Convolve image $f(r, c)$ with a Gaussian function to get smooth image $f^{\wedge}(r, c)$. $\mathrm{f}^{\wedge}(\mathrm{r}, \mathrm{c})=\mathrm{f}(\mathrm{r}, \mathrm{c})^{*} \mathrm{G}(\mathrm{r}, \mathrm{c}, 6)$

- Apply first difference gradient operator to compute edge strength then edge magnitude and direction are obtain as before.

- Apply non-maximal or critical suppression to the gradient magnitude.

- Apply threshold to the non-maximal suppression image.

Unlike Roberts and Sobel, the Canny operation is not very susceptible to noise. If the Canny detector worked well it would be superior. 
International Journal of Computer Science \& Information Technology (IJCSIT) Vol 3, No 6, Dec 2011

\section{EXPERIMENTAL RESULT}

This section presents the relative performance of various edge detection techniques such as Roberts edge detector, Sobel Edge Detector, Prewitt edge detector, Kirsch, Robinson, MarrHildreth edge detector, LoG edge detector and Canny Edge Detector.

The edge detection techniques were implemented using MATLAB R2009a, and tested with an image (Bharathiar University). The objective is to produce a clean edge map by extracting the principal edge features of the image. The original image and the image obtained by using different edge detection techniques are given in figure.

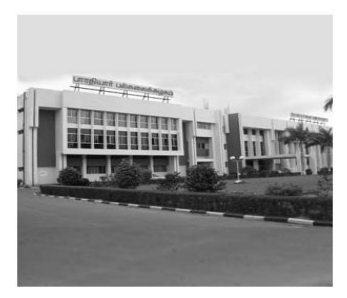

Original

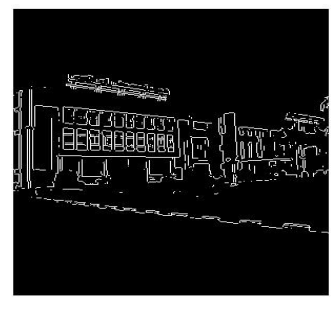

Prewitt

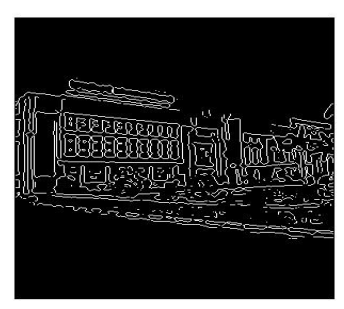

Marr-Hildreth

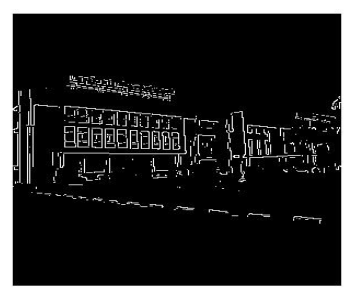

Roberts

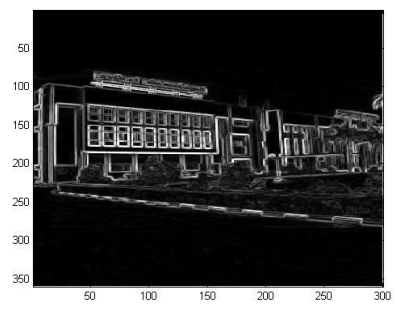

Kirsch

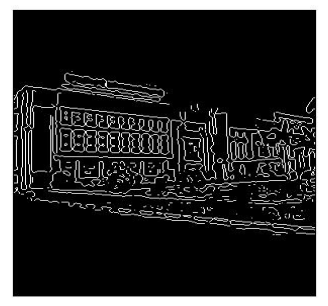

LoG

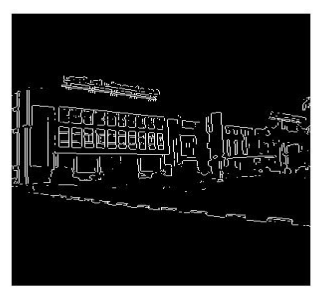

Sobel

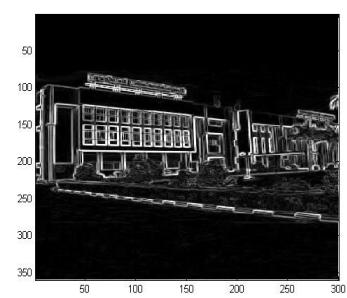

Robinson

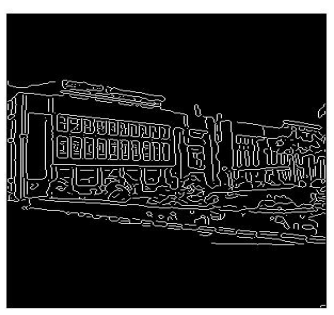

Canny

Figure: Original Image with the result of various edge detection techniques

Roberts, Sobel and Prewitt results actually deviated from the others. Marr-Hildreth, LoG and Canny produce almost same edge map. Kirsch and Robinson edge maps are almost same. It is observed from the figure, Canny result is superior by far to the other results. 
International Journal of Computer Science \& Information Technology (IJCSIT) Vol 3, No 6, Dec 2011

\section{CONCLUSION}

In the discipline of computer vision, image processing is a quickly moving field. Its growth has been fueled by technological advances in digital imaging, computer processors and mass storage devices. In this paper an attempt is made to review the edge detection techniques which are based on discontinuity intensity levels. The relative performance of various edge detection techniques is carried out with an image by using MATLAB software. It is observed from the results Marr-Hildreth, LoG and Canny edge detectors produce almost same edge map. Canny result is superior one when compared to all for a selected image since different edge detections work better under different conditions. Even though, so many edge detection techniques are available in the literature, since it is a challenging task to the research communities to detect the exact image without noise from the original image.

\section{REFERENCES}

[1] Canny, J. F (1983) Finding edges and lines in images, Master's thesis, MIT. AI Lab. TR-720.

[2] Canny, J. F (1986) “A computational approach to edge detection”, IEEE Transaction on Pattern Analysis and Machine Intelligence, 8, 679-714.

[3] Courtney. P \& N. A. Thacker (2001) "Performance Characterization in Computer Vision: The Role of Statistics in Testing and Design", Chapter in: "Imaging and Vision Systems: Theory, Assessment and Applications", Jacques Blanc-Talon and Dan Popescu (Eds.), NOVA Science Books.

[4] Hanzi Wang (2004) Robust Statistics for Computer Vision: Model Fitting, Image Segmentation and Visual Motion Analysis, Ph.D thesis, Monash University, Australia.

[5] Huber, P.J. (1981) Robust Statistics, Wiley New York.

[6] Kirsch, R. (1971) "Computer determination of the constituent structure of biological images", Computers and Biomedical Research, 4, 315-328.

[7] Lakshmi,S \& V.Sankaranarayanan (2010) "A Study of edge detection techniques for segmentation computing approaches", Computer Aided Soft Computing Techniques for Imaging and Biomedical Applications, 35-41.

[8] Lee, K.. M, Meer, P. \& et al. (1998) "Robust Adaptive Segmentation of Range Images", IEEE Trans. Pattern Analysis and Machine Intelligence, 20(2), 200-205.

[9] Marr, D \& E. Hildreth (1980) “Theory of edge detection”, Proc. Royal Society of London, B, 207, 187-217.

[10] Marr, D(1982) Vision, Freeman Publishers.

[11] Marr, P \& Doron Mintz, D. \& et al. (1991) "Robust Regression for Computer Vision: A Review”, International Journal of Computer Vision, 6(1), 59-70.

[12] Orlando, J, Tobias \& Rui Seara (2002) "Image Segmentation by Histogram Thresholding Using Fuzzy Sets”, IEEE Transactions on Image Processing, Vol.11, No.12, 1457-1465.

[13] Punam Thakare (2011) “A Study of Image Segmentation and Edge Detection Techniques", International Journal on Computer Science and Engineering, Vol 3, No.2, 899-904.

[14] Rafael C. Gonzalez, Richard E. Woods \& Steven L. Eddins (2004) Digital Image Processing Using MATLAB, Pearson Education Ptd. Ltd, Singapore. 
International Journal of Computer Science \& Information Technology (IJCSIT) Vol 3, No 6, Dec 2011

[15] Ramadevi, Y \& et al (2010) "Segmentation and object recognition using edge detection techniques”, International Journal of Computer Science and Information Technology, Vol 2, No.6, 153-161.

[16] Roberts, L (1965) "Machine Perception of 3-D Solids", Optical and Electro-optical Information Processing, MIT Press.

[17] Robinson. G (1977) "Edge detection by compass gradient masks", Computer graphics and image processing, 6, 492-501.

[18] Rousseeuw, P. J \& Leroy, A (1987) Robust Regression and outlier detection, John Wiley \& Sons, New York.

[19] Senthilkumaran. N \& R. Rajesh (2009) "Edge Detection Techniques for Image Segmentation A Survey of Soft Computing Approaches", International Journal of Recent Trends in Engineering, Vol. 1, No. 2, 250-254.

[20] Sowmya. B \& Sheelarani. B (2009) "Colour Image Segmentation Using Soft Computing Techniques”, International Journal of Soft Computing Applications, Issue 4, 69-80.

[21] Umesh Sehgal (2011) "Edge detection techniques in digital image processing using Fuzzy Logic", International Journal of Research in IT and Management, Vol.1, Issue 3, 61-66.

[22] Yu, X, Bui, T.D. \& et al. (1994) "Robust Estimation for Range Image Segmentation and Reconstruction”, IEEE trans. Pattern Analysis and Machine Intelligence, 16 (5), 530-538. 